\title{
Patient safety in genomic medicine: an exploratory study
}

\author{
Diane M. Korngiebel, DPhil', Stephanie M. Fullerton, DPhil² and Wylie Burke, MD, PhD²
}

\begin{abstract}
Purpose: Concerns about patient safety and the potential for medical error are largely unexplored for genetic testing, despite the expansion of test use. In this preliminary qualitative study, we sought the views of genetics professionals about error and patient safety concerns in genomic medicine and factors that might mitigate them.

Methods: Twelve semistructured interviews with experienced genetics professionals were conducted. Transcripts were analyzed using selective coding for issues related to error definition, mitigation, and communication. Additional thematic analysis captured themes across content categories.
\end{abstract}

Results: Key informants suggested that the potential for adverse events exists in all phases of genetic testing, from ordering to analysis, interpretation, and follow-up. A perceived contributor was lack of physician knowledge about genetics, resulting in errors in test ordering and interpretation. The limitations and uncertainty inherent to rapidly evolving technology were also seen as contributing factors. Strategies to prevent errors included physician education, availability of genetic experts for consultation, and enhanced communication such as improved test reports and electronic decision support.

Conclusion: Genetic testing poses concerns for patient safety due to errors and the limitations of current tests. As genomic tests are integrated into medical care, anticipating and addressing patient safety concerns identified by these key informants will be crucial.

Genet Med advance online publication 24 March 2016

Key Words: genetic testing; genomic medicine; medical error; patient safety; precision medicine

\section{INTRODUCTION}

Genetic tests are increasing in scope and complexity, bringing with them an array of challenges to the responsible implementation of genomic medicine. ${ }^{1-4}$ Although only limited attention has been directed to patient safety as genomic testing expands to diverse clinical settings, current available evidence is concerning. A variety of adverse outcomes related to errors in cancer-related genetic testing have been described in reports collected by members of the National Society of Genetic Counselors. ${ }^{5-7}$ Another study reported that patients were sometimes offered inappropriate care based on misinterpretation of test results, such as prophylactic mastectomy in response to a variant of unknown significance (VUS) in a BRCA1 or BRCA2 gene. ${ }^{8}$ Other recent work found that $25 \%$ of clinical genetic tests performed in diagnostic laboratories were incorrectly ordered, leading to increased costs. ${ }^{9}$ These and related anecdotes suggest the need to more systematically investigate the potential for error in clinical genomic testing in an effort to identify and proactively address common contributing factors.

As a first step toward examining implications for patient safety in genomic medicine, we conducted a pilot study, exploratory in nature, that used qualitative methods to investigate the views of medical genetics professionals. In beginning with geneticists, we reasoned that those with extensive experience with genetic test ordering, interpretation, and follow-up would more likely have observed or heard about patient safety lapses and, perhaps more importantly, would be well positioned to anticipate ways in which current practices could be adapted to minimize mistakes in the future. Effective management of medical error in other clinical contexts has been based on designing systems of health-care delivery that anticipate and, whenever possible, prevent errors and their associated adverse outcomes ${ }^{10,11}$ as well as on proactively training personnel to identify patient safety issues and their causes. ${ }^{12,13}$ In the spirit of an exploratory investigation, we invited respondents to identify what they would recognize as harms, allowing the discussion to encompass frank medical errors as well as unanticipated adverse outcomes or negative patient reactions that might not generally be associated with patient safety.

\section{Recruitment}

\section{METHODS AND MATERIALS}

Twelve key informants, eight male and four female, from the Puget Sound region in Washington State were interviewed. Initial participants were identified by professional roles in medical genetics practice, including clinical genetics, laboratory-based genetics, and genetic counseling; additional interview participants were identified via "snowball sampling" (i.e., initial interviewees' recommendations of other key informants). Each participant had more than 15 years of experience as a genetics professional.

\section{Data collection}

Interviews were conducted in person or by telephone using a semistructured interview guide designed to elicit participants'

${ }^{1}$ Biomedical Informatics and Medical Education, University of Washington, Seattle, Washington, USA; ${ }^{2}$ Bioethics and Humanities, University of Washington, Seattle, Washington, USA. Correspondence: Stephanie M. Fullerton (smfllrtn@uw.edu) 
views of medical error in medical genetics, reflections on specific testing experiences, and thoughts regarding implications of these experiences for future clinical practice, including strategies to help prevent future errors (Table 1).

Each interview lasted between 45 and $90 \mathrm{~min}$. Interviews were recorded and transcribed, and the transcripts were deidentified; study identification numbers were assigned to participants to protect confidentiality. The study was approved by the University of Washington Institutional Review Board, and all participants provided informed consent.

\section{Data analysis}

All transcripts were uploaded into the qualitative analysis software Atlas.ti 7 for coding and content analysis. A codebook was created by the study team that incorporated a priori categories related to when genetic testing occurs (e.g., at the time of test ordering) and included communication issues as a theme drawn from the patient safety literature. Transcripts were coded by a single analyst with review by other members of the study team. Modified grounded theory was used to accommodate a priori areas of focus while allowing for identification of additional themes not anticipated by the original codebook. $^{14,15}$

\section{RESULTS}

Key informants described their observations of errors in genetic testing, their assessment of related testing challenges that might lead to adverse outcomes, and their ideas for practice changes that might enhance patient safety and prevent future errors.

\section{Errors described by key informants}

Respondents noted that errors and adverse events occurred during all phases of genetic testing, from test ordering to analysis, test interpretation, and clinical follow-up.

Incorrect or inappropriate test ordering. Inappropriate test ordering was noted as a significant issue, with errors often attributed to the inexperience of the test orderer. Mistakes noted included ordering a test when the diagnosis could be made clinically or ordering the wrong test from the wide array of available options, including single-gene tests, gene panels of varying sizes, and whole-exome and related tests. In the latter context, one respondent described a situation in which a patient with clear symptoms of Charcot-Marie-Tooth disease was seen by a neurologist with limited expertise in genetics. The neurologist ordered a $\$ 16,000$ panel test instead of a much less costly test for the PMP22 duplication that accounts for most cases of the disease:

"the PMP22 duplication came back positive. So there was a $\$ 15,000$ mismatch in cost and it turned out the patient's insurance wouldn't cover it... after all the negotiations, the patient got stuck with a $\$ 10,000$ bill. The whole thing could have been solved for $\$ 800$ or $\$ 900$." (Participant 10)

Another example involved failure to proceed with additional testing after a normal $B R C A 1 / 2$ result, for a patient with earlyonset breast cancer, an error that delayed disease surveillance and follow-up testing of family members:

Table 1 Representative domains and sample questions from the interview guide

\begin{tabular}{|c|c|}
\hline Domain & Sample questions \\
\hline Medical error in medical genetics & $\begin{array}{l}\text { - How does medical error happen? What does it look like? } \\
\text { - How often do such errors occur? } \\
\text { - What contributes to these errors? } \\
\text { - How are errors handled? } \\
\text { - Are some kinds of errors more likely with sequence-based tests? }\end{array}$ \\
\hline $\begin{array}{l}\text { Unanticipated outcomes in medical } \\
\text { genetics }\end{array}$ & $\begin{array}{l}\text { - Are there other unanticipated outcomes that patients might experience as a result of genetic testing? } \\
\text { - What role do uncertain results (e.g., variants of uncertain significance) play in the potential for } \\
\text { unanticipated (adverse) outcomes? }\end{array}$ \\
\hline Specific experiences & $\begin{array}{l}\text { - Have you observed an unanticipated outcome that could be attributed to a medical error? Please describe } \\
\text { what happened. } \\
\text { - Have you observed an unanticipated outcome that was not due to a medical error? Please describe what } \\
\text { happened. }\end{array}$ \\
\hline Implications for practice or policy & $\begin{array}{l}\text { - Are you aware of any policies or procedures to reduce the likelihood of unanticipated outcomes and/or } \\
\text { guide how errors are handled? } \\
\text { - If yes, what are they? What effect, if any, have these had on your practice? } \\
\text { - If no, why do you think that is? } \\
\text { What steps would you recommend for proactively addressing the possibility of error in medical genetic } \\
\text { practice? }\end{array}$ \\
\hline
\end{tabular}


"She was told that [it] wasn't genetic, don't worry about it. She developed another cancer. We would have done $B R C A 1$ and 2 first- - so that's not an error-but we wouldn't have stopped...She's been told it's not genetic by her oncologist, don't worry about your sisters, your children-and she has a p53 mutation. She's devastated." (Participant 1)

Although lack of knowledge on the part of nongeneticists was identified as an important problem, respondents also noted presumed errors involving genetics professionals. For example, one participant had seen an Ashkenazi woman whose mother had a history of two breast cancers and ovarian cancer. According to the key informant, this patient had been told by another genetics professional "not to worry about it [i.e., a familial predisposition]. And I'm sorry, that's clearly an error" (Participant 1). In this case, the participant reported that the patient's brother had died of pancreatic cancer and her mother was subsequently sent for genetic testing and discovered to have a BRCA2 mutation, confirming the participant's impression that the initial advice against testing had been a mistake.

Errors of analysis. Errors in the process of laboratory analysis were less frequently described and were attributed to sample mishandling or the testing process itself. For example, one participant described an observed inconsistency between a functional test and a genetic test:

"So I had this discrepancy here between the functional results saying she does have HPS [Hermansky-Pudlak Syndrome] and the molecular result which says she doesn't have it...So I was going to call [the laboratory geneticist who performed the test]... and say what other kind of testing is there? Can we do something else to confirm this... and then when I'm talking to him he decides to pull the slides and review them again... and then he tells me he made a mistake. Sample mix-up." (Participant 5)

Other problems with laboratory analysis were related to inherent limitations of the testing process. For example, one respondent cited the issue of read depth (i.e., the redundancy of sequence information generated from a given test) as a potential source of erroneous results from next-generation sequencing: "I can tell you that you can be led astray...very easily if you're not paying attention to the...implied precision of the test" (Participant 3). Erroneous results were also sometimes related to unrecognized problems with a test assay, including the use of inadequate reference databases. As one key informant explained:

"We missed a variant that was there because we were using information for a database about the primers that we were using that would tell us that the results should be okay. And the database was wrong, and so we missed this variant and fortunately it was picked up [by someone else]. I mean there wasn't a bad outcome but there could have been." (Participant 8)

Incorrect test interpretation or follow-up. Finally, errors of test interpretation and associated follow-up were described by respondents; in many cases, these involved variants of unknown significance (VUS). One key informant, for example, described the misdiagnosis of a patient due to a nonspecialist interpreting a VUS result as a definitive genetic finding:

"We will see a patient from cardiology—and [we] actually have clearly told the patient and documented this is a VUS - and they go back and see the cardiologist and [the cardiologist says] in the note they have a mutation and therefore they have this disease." (Participant 5)

In other cases, key informants described clinical management pursued in the face of a VUS finding that may be inappropriate or unnecessary, particularly if the uncertain variant is subsequently reclassified as benign:

"We certainly see a reasonable fraction of women who have BRCA1 and 2 VUSes who go on to have mastectomies and oophorectomies and is that a medical error? Is that misinterpretation of the test?... But most of the VUSes-and we tell the patients this-most of the VUSes turn out to be benign. And we do signal if there's something about where it is, if it's highly conserved or very negative change, that we think may, or may be more likely to not, be benign. But really for things that we thought that were likely to be benign, we see people getting surgery and that suggests to me that there's not a complete understanding of what the VUS means." (Participant 1)

\section{Adverse outcomes due to other testing challenges}

Although participants described experiences involving errors in the testing process, they also described adverse outcomes without attributing them to clinician error, and they sometimes found it difficult to define what constitutes a testing error. Key informants noted adverse outcomes after an appropriate and correctly performed testing process and also when test results were suboptimal or uncertain as a result of the current state of genomic technology. Several key informants, for example, noted that adverse outcomes can occur because of the nature of the information generated by a test:

"You know, if we give people the correct information and that correct information is devastating to them and they kill themselves, that's an adverse outcome but it wasn't because you did the wrong thing." (Participant 9)

However, in other cases, there was more ambiguity about the role of clinical practice, including how test information is transmitted, in adverse outcomes. One respondent described a situation in which a patient was given a diagnosis of Huntington 
disease over the phone, without support or counseling, and subsequently committed suicide:

"I don't know what's the cause and effect...Maybe we couldn't have prevented it... But we might have had a better chance...That's an error...And maybe it's not an error, but it's a harm." (Participant 4)

Limitations in the technology or knowledge available to the clinician can lead to insufficient information to address the clinical question. In this regard, limited test sensitivity and evolving technology both represent important challenges for genomic medicine but also reflect the uncertainty already inherent in clinical practice:

"One limiting factor is the diagnostic acumen in our knowledge of clinical disease and recognition of clinical disease and how that correlates with the vast new information that one gets from whole-genome sequencing and variations and how one makes that 1:1 correlation. So there's errors on both sides of that spectrum... Well, it [genome technology] increased the ambiguity of clinical diagnosis, but there's ambiguity in clinical diagnosis...I mean, all you have to do is look at the natural history of medicine. How many new diagnoses do we have today versus even 20 years ago?" (Participant 7 )

Further complicating matters is that as tests improve over time, previous test results may become erroneous in retrospect. One respondent noted:

"You have the individual who has what you think is a clinical disorder; you do the mutational testing on them. The mutational testing is negative, but you know the sensitivity of that test is less than $100 \%$. You probably don't modify much of what you tell that patient except you can't offer them, for example, prenatal diagnosis. Let's say you find out later that there's a new technology and it can find a mutation in the intron which was missed before...So then, is it an error because you haven't gone back and informed them about something new? It didn't make what you did at the time wrong." (Participant 9)

\section{Mitigation strategies}

Our participants thought that some errors, such as mix-ups in laboratory samples, are not specific to genetics and can be addressed by patient safety measures already in place. However, they also suggested a number of mitigation strategies that will be important for genomic medicine, including physician education and enhanced genetics referral, as well as communication strategies such as improved test reports and decision tools embedded in electronic medical records.

Assisting clinicians with limited genetics expertise. Informants identified lack of genetics expertise as a contributor to errors at several points in the testing process. Therefore, several participants suggested physician education as a key mitigation strategy. They noted the importance of efforts to strengthen teaching of genetics in medical school curricula and to develop continuing education and in-services for physicians in practice.

"We did a quality improvement session for them...one point I made is that although genetic testing has greatly increased among neurologists-and for good reason because many neurologic conditions have a genetic basisthey don't have a formal requirement for genetic training in their residency. Right? They have to learn neuropathology, neuroradiology, electrophysiology, a lot of things, and they can do genetics as an elective, but it's not systematic, it's not organized, it's not a residency in genetics." (Participant 5)

Nevertheless, participants also noted that education alone was unlikely to be sufficient. Related strategies included increased availability of experts for consultation and improvement in communication across health-care sectors.

"You certainly can't train physicians who are already out of medical school many years to get all of these in great detail. Medical practice is so busy and so overwhelming... I think we need people like genetic counselors, we need more of them who are around and available and used by both the labs and the clinician to help sort these things out." (Participant 10)

One participant noted the value of collaboration between clinicians with phenotypic and genetics expertise, that is,

"having the people that understand the phenotype whatever it is, lung, gut, heart, whatever, have a handle on genetics or maybe partner with a geneticist who...is really interested in heart and the heart person is really interested in genetics. You're going to get a lot further.' (Participant 3 )

In the absence of local experts, remote access to expertise was also suggested as a way forward: "A physician doesn't necessarily have to be physically there. It sounds to me like an ideal opportunity for telegenetics." (Participant 12)

Improved reporting of test findings. Participants noted that both laboratory reports and letters from medical genetics to referring clinicians represent important opportunities to improve communication:

"Especially in genetics...our clinic notes, and letters, are like educational pieces. We get criticized for how long our notes are and letters, but I think part of it is that we're trying to educate the community." (Participant 4 )

However, the optimal balance between sufficient and excessive information ("overwhelming them with data") was recognized as difficult to achieve: 


\section{ORIGINAL RESEARCH ARTICLE}

\begin{abstract}
"Reports [have] been a problem since the beginning. I mean, I can remember the days when we had long conversations with our people in the genetic testing lab...I mean, how do we format these reports? They err on both sides. Sometimes they err on being too short...Then there are other reports that are way, way too detailed. They're three pages long and they go on and on with the message and references... and the physician scratches his or her head trying to figure out, 'Now where is the answer?"' (Participant 10)
\end{abstract}

One participant emphasized the responsibility of the laboratory to guide accurate interpretation, providing evidence to support the classification of a gene variant as pathogenic, benign, or VUS:

\begin{abstract}
"They did the test. They got paid for the test and they got paid for the results and these results have to be interpreted. You can't just send me back a number...make labs accountable for the information they give." (Participant 9)
\end{abstract}

Similarly, our participants emphasized the importance of clearly stating limitations, and resulting uncertainty, in genetic test reports, whether due to limited evidence for variant interpretation or to inherent limitations in analytic methods.

Alerts in the electronic medical record. Finally, at least some participants saw leveraging the electronic medical record as an important way to enhance communication and provide expert knowledge.

"I really think we need clinical decision support for the interpretation of these tests. So, not just what we write in our genetics note that says this is what we found and this is what we think should be done but decision support that comes up and alerts the primary care doctor if they're ordering a test that may not be appropriate or that they are not ordering the screening tests that would be appropriate. I think that there aren't enough geneticists out there to follow these people. They have to return to their primary care providers and that decision support is going to be extremely useful." (Participant 1)

However, developing effective decision support was seen as a challenge:

"[The difficulty] is finding a mechanism for introducing this type of information in a way that is easy to use, easy to understand, and then not prone to error. And I'm always amazed that medical record systems have a hard time with this...And we've been hearing the stories of different units, finding it very, very difficult. They're exploring different ways in which the information is communicated, alert systems and the like but until-you know, they're experimenting. They're playing around trying to find out what is the best model, what is the best system, best way to communicate the information. Until that gets sorted out, there is the potential for error in there." (Participant 2)

\section{DISCUSSION}

These data confirm and extend previous reports suggesting that patient safety is a potential concern in genomic medicine. ${ }^{7-9,16}$ Prompted by the interview protocol to consider both frank medical errors and unanticipated adverse outcomes, our key informants-all experienced genetics professionals-offered numerous examples of testing-related errors they had observed and also noted that an adverse or unexpected outcome from testing may or may not be due to an error, such as patient responses to the return of life-altering test results (e.g., suicide after a diagnosis of Huntington disease). ${ }^{17-19}$ These observations point to opportunities and challenges in addressing patient safety as genetic and genomic tests become more widely integrated into diverse forms of medical care.

Our key informants described examples of errors in test ordering, analysis, interpretation, and follow-up, suggesting that all phases of genetic testing are subject to error and/or adverse events. In certain cases, frank errors of clinical judgment were noted-such as the failure to pursue genetic testing in the face of a suggestive family history that resulted in additional disease burden for the patient and a lost opportunity to identify at-risk relatives. In many other cases, however, the examples suggested an interplay between diverse and increasingly complex test options (e.g., choices of test platform to use, rapidly shifting changes in reference databases) and the relative inexperience of clinicians ordering tests. Concerns about the lack of genetics knowledge among health-care providers now ordering genetic tests or determining posttest follow-up is in keeping with calls for improved genetics education, including requests for more education from primary-care providers themselves. ${ }^{20-22}$ Although recognition of this issue is important, experience from the patient safety movement also suggests a need to avoid a "shame and blame" approach in which errors are simply attributed to clinicians lacking expertise or otherwise making practice errors. ${ }^{10}$ Instead, systems-based approaches that minimize errors in test ordering and interpretation, which are already being examined elsewhere, ${ }^{23}$ will be needed.

We invited our key informants to think prospectively with us about strategies that could be implemented to anticipate and forestall errors of the kind that they described. Many of the ideas generated in this portion of our interview focused on ways to assist nonexpert clinicians with test ordering and interpretation. Suggestions included strategies to improve clinician access to genetics expertise, crafting of genetic test reports in ways that might inform and guide nongeneticists, and the use of decision tools within electronic medical records to provide point-of-service guidance.

Even as these suggestions were being offered, however, key informants noted significant barriers that would need to be addressed, including ensuring timely availability of genetic counseling and related expertise, balancing the 
need to educate but not overwhelm recipients of genetic test reports, and strategic use of clinical decision support that would facilitate, not complicate, test ordering. A subset of our respondents expressed skepticism about the ability to eliminate all potential adverse events, noting the near inevitability of some adverse outcomes even in the absence of clinical error.

The consistent concern of respondents about the role of nonexperts in patient safety-related considerations indirectly highlights the central role of teamwork and team-related communication in mediating patient safety. A variety of approaches to addressing professional silos and organizational barriers to cross-disciplinary coordination and communication have proven effective in other clinical settings, ${ }^{24-26}$ such as the institution of checklist interventions that ensure that all members of a surgical team know and understand one another's role in the planned procedure. ${ }^{27-30}$ Silos created by structural limitations (e.g., independent departments, clinics, health-care systems, laboratories, and commercial entities) can contribute to reduced communication and limit the ability to make appropriate decisions or take suitable action. ${ }^{31}$ In applying this concept to genetic testing, the challenge is to ensure effective functioning of what is, in many cases, a distributed health-care team, whose members may work in different clinical settings and sometimes different cities, providing care to a patient and family members over an expanded period of time. Our participants offered some thoughts about how to overcome communication barriers, but additional research will be needed to determine how best to support such teamwork in the safe and effective use of genetic and genomic tests.

These preliminary findings underscore the need for more sustained, and systematic, investigation of potential patient safety lapses in genomic medicine. The main limitation of the current study is the generalizability of these findings beyond the interviewed cohort, which was small and composed exclusively of genetics professionals sampled from a single geographic region. In addition, the lack of viewpoints from a wider group of professionals who use or interact with genetic tests probably also affected saturation, which was not achieved. This preliminary study identified specific topics and concerns that can help guide subsequent research, for example, the need to involve nongenetics clinicians in patient safety explorations of genomic medicine delivery, to explore interactions between genetics professionals and other clinicians, and to investigate teamwork more generally. Additional qualitative inquiry of issues related to error and patient safety in genomic medicine is urgently needed to explore the views of collaborating nongeneticist clinicians who order genetic tests, as well as the patients they serve. Only then will we be able to craft effective interventions with the capacity to mitigate potential error in this rapidly expanding domain of clinical practice.

\section{ACKNOWLEDGMENTS}

We acknowledge Thomas H. Gallagher and the UW Medicine Center for Scholarship in Patient Care Quality and Safety for their support and guidance in the development of this pilot investigation.
The content is solely the responsibility of the authors and does not necessarily represent the official views of the National Institutes of Health or the authors' affiliated institutions.

\section{DISCLOSURE}

The authors' work has been funded by the NIH National Human Genome Research Institute: P50 HG03374 (W.B.), R21 HG007578 (S.M.F.), and K01 HG008180 (D.M.K.). The authors declare no other conflicts of interest.

\section{REFERENCES}

1. Burke W, Korngiebel DM. Closing the gap between knowledge and clinical application: challenges for genomic translation. PLoS Genet 2015;11:e1004978.

2. Manolio TA, Chisholm RL, Ozenberger $B$, et al. Implementing genomic medicine in the clinic: the future is here. Genet Med 2013;15:258-267.

3. Levy BP, Chioda MD, Herndon D, et al. Molecular testing for treatment of metastatic non-small cell lung cancer: how to implement evidence-based recommendations. Oncologist 2015;20:1175-1181.

4. Abul-Husn NS, Owusu Obeng A, Sanderson SC, Gottesman O, Scott SA. Implementation and utilization of genetic testing in personalized medicine. Pharmgenomics Pers Med 2014;7:227-240.

5. Brierley KL, Campfield D, Ducaine W, et al. Errors in delivery of cancer genetics services: implications for practice. Conn Med 2010;74:413-423.

6. Brierley KL, Blouch E, Cogswell W, et al. Adverse events in cancer genetic testing: medical, ethical, legal, and financial implications. Cancer J 2012;18:303-309.

7. Bonadies DC, Brierley KL, Barnett RE, et al. Adverse events in cancer genetic testing: the third case series. Cancer J 2014;20:246-253.

8. Murray ML, Cerrato F, Bennett RL, Jarvik GP. Follow-up of carriers of BRCA1 and BRCA2 variants of unknown significance: variant reclassification and surgical decisions. Genet Med 2011;13:998-1005.

9. Miller CE, Krautscheid P, Baldwin EE, et al. Genetic counselor review of genetic test orders in a reference laboratory reduces unnecessary testing. Am J Med Genet A 2014;164A:1094-1101.

10. Reason J. Human error: models and management. BMJ 2000;320:768-770.

11. Broder JS, Fox JW, Milne J, Theiling BJ, White A. Heimlich valve orientation error leading to radiographic tension pneumothorax: analysis of an error and a call for education, device redesign and regulatory action. Emerg Med J;e-pub ahead of print 3 November 2015.

12. Trinkley KE, Van Matre ET, Mueller SW, Page RL 2nd, Nair K. Perceived benefit of teaching patient safety to pharmacy students by integrating classroom teaching with introductory (IPPE) visits. J Pharm Pract; e-pub ahead of print 30 October 2015.

13. Frey B, Doell C, Klauwer D, et al. The morbidity and mortality conference in pediatric intensive care as a means for improving patient safety. Pediatr Crit Care Med 2016;17:67-72.

14. Glaser BG, Strauss AL. The Discovery of Grounded Theory: Strategies for Qualitative Research. Aldine de Gruyter: New York, 1967.

15. Ryan GW, Bernard HR. Techniques to identifying themes. Field Methods 2003;15:85-109.

16. ARUP Laboratories. Value of Genetic Counselors in the Laboratory. University of Utah: Salt Lake City, UT, 2011

17. Crozier S, Robertson N, Dale M. The psychological impact of predictive genetic testing for Huntington's disease: a systematic review of the literature. J Genet Couns 2015;24:29-39.

18. Godino L, Turchetti D, Jackson L, Hennessy C, Skirton H. Impact of presymptomatic genetic testing on young adults: a systematic review. Eur J Hum Genet; e-pub ahead of print 15 July 2015.

19. Lam RW, Bloch $M$, Jones BD, et al. Psychiatric morbidity associated with early clinical diagnosis of Huntington disease in a predictive testing program. J Clin Psychiatry 1988;49:444-447.

20. Christensen KD, Vassy JL, Jamal L, et al. Are physicians prepared for whole genome sequencing? a qualitative analysis. Clin Genet 2016;89:228-234.

21. Mikat-Stevens NA, Larson IA, Tarini BA. Primary-care providers' perceived barriers to integration of genetics services: a systematic review of the literature. Genet Med 2015;17:169-176.

22. Kaye C, Korf B. Genetic literacy and competency. Pediatrics 2013;132(suppl 3):S224-S230.

23. Bowie P, Forrest E, Price J, et al. Good practice statements on safe laboratory testing: a mixed methods study by the LINNEAUS collaboration on patient safety in primary care. Eur J Gen Pract 2015;21(suppl):19-25. 


\section{ORIGINAL RESEARCH ARTICLE}

24. Weller J, Boyd M, Cumin D. Teams, tribes and patient safety: overcoming barriers to effective teamwork in healthcare. Postgrad Med $J 2014$.

25. Vertino KA. Evaluation of a TeamSTEPPS @ initiative on staff attitudes toward teamwork. J Nurs Adm 2014;44:97-102.

26. Haynes J, Strickler J. TeamSTEPPS makes strides for better communication. Nursing 2014;44:62-63.

27. Date DF, Sanfey H, Mellinger J, Dunnington G. Handoffs in general surgery residency, an observation of intern and senior residents. Am J Surg 2013;206:693-697.
28. Fudickar A, Hörle K, Wiltfang J, Bein B. The effect of the WHO Surgical Safety Checklist on complication rate and communication. Dtsch Arzteb/ Int 2012;109:695-701.

29. Gillespie BM, Marshall A. Implementation of safety checklists in surgery: a realist synthesis of evidence. Implement Sci 2015;10:137.

30. Treadwell JR, Lucas S, Tsou AY. Surgical checklists: a systematic review of impacts and implementation. BMJ Qual Saf 2014;23:299-318.

31. Hughes AM, Salas E. Hierarchical medical teams and the science of teamwork. Virtual Mentor 2013;15:529-533. 